\title{
TNF superfamily member TL1A elicits type 2 innate lymphoid cells at mucosal barriers
}

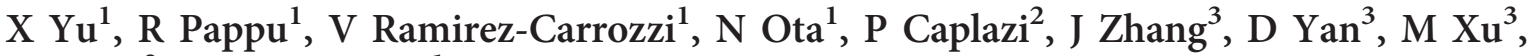 \\ WP Lee ${ }^{3}$ and JL Grogan ${ }^{1}$
}

Immune responses at mucosal barriers are regulated by innate type 2 lymphoid cells (ILC2s) that elaborate effector cytokines interleukins 5 and 13 (IL5 and IL13). IL25 and IL33 are key cytokines that support ILC2s; however, mice deficient in these pathways retain some functional ILC2s. Analysis of human and murine cells revealed that ILC2s highly express tumor necrosis factor (TNF)-receptor superfamily member DR3 (TNFRSF25). Engagement of DR3 with cognate ligand TL1A promoted ILC2 expansion, survival, and function. Exogenous protein or genetic overexpression of TL1A activated ILC2s independent of IL25 or IL33. $\mathrm{Dr}^{-1-}$ mice failed to control gut helminthic infections, and failed to mount ILC2 responses in the lung after nasal challenge with papain. Our data demonstrate a key role for TL1A in promoting ILC2s at mucosal barriers.

\section{INTRODUCTION}

Innate lymphoid cells (ILCs) are a recently described group of innate immune cells that regulate immunity, inflammation, and tissue repair. Innate immune responses provide first-line immunity to invading pathogens. Although only a small fraction of the total immune cell population, they are found throughout the immune system, particularly at epithelial barriers, suggesting they have a critical role in maintaining the integrity of the skin, airways, and intestine. ILC subsets have been defined based on distinct pattern of transcription factor expression, cell surface markers, and cytokines, much like $\mathrm{CD}^{+}{ }^{+} \mathrm{T}$ helper (Th) cell subsets. As such, ILC1, ILC2, and ILC3 produce the same signature cytokines as Th1, Th2, and Th17/Th22 cells, respectively. The fact that these ILC subsets elaborate different cytokines yet lack rearranged antigenspecific receptors implicate them as evolutionary precursor of cells of the adaptive immune system that may facilitate crosstalk between the innate and adaptive arms of the immune response. ${ }^{1,2}$

ILC2s in particular play a critical role in protection against helminthic infections, in tissue repair after influenza virus infection, and contribute to the pathophysiology of allergic inflammation and asthma. ${ }^{3-8}$ Interleukin-25 (IL25) and IL33 are two key cytokines that support ILC2 differentiation and function. ${ }^{6,9}$ All ILCs lack lineage markers $\left(\mathrm{Lin}^{-}\right.$) but express the lymphoid progenitor marker IL7 receptor $\alpha$ chain (IL7R $\alpha$; $\mathrm{CD} 127)$ and the cytokine common gamma $(\gamma \mathrm{c})$ receptor chain, and require IL7 for development. ${ }^{10}$ In addition, ILC2s express the receptor for IL33, T1/ST2, and the receptor for IL25, IL17RB. ${ }^{2}$ ILC2s were originally identified in Rag-deficient mice after stimulation with IL25 or IL33, as the cellular source of IL5 and IL13. ${ }^{11}$ IL25R/IL33R doubly deficient mice have a severely impaired response to helminth infection. Interestingly, although mice deficient for IL25 or IL33 or the receptors for IL25 or IL33 have impaired ILC2 responses, ${ }^{12-14}$ some functional ILC2s still remain, indicating the existence of additional pathways that support ILC2 biology. As ILC2s are found predominantly at epithelial barriers, which when perturbed elicit a flux of inflammatory factors including IL25 and IL33, suggest that other inflammatory mediators may regulate their generation and function. Indeed, it has been recently reported that ILC2s found in the skin respond synergistically to thymic stromal lymphopoietin (TSLP) and IL33. ${ }^{15}$

TL1A belongs to tumor necrosis factor (TNF) superfamily (TNFSF), a family of cytokines that are evolutionally conserved and play important roles in regulating both innate and adaptive immune cells. ${ }^{16}$ TL1A is highly expressed by activated myeloid cells and stressed epi- and endothelial cells in the gut and lung barriers. Genome-wide association studies have identified

\footnotetext{
${ }^{1}$ Department of Immunology, Genentech, South San Francisco, California, USA. 'Department of Pathology, Genentech, South San Francisco, California, USA and ${ }^{3}$ Department of Translational Immunology, Genentech, South San Francisco, California, USA. Correspondence: JL Grogan (jgrogan@gene.com)
} 
single-nucleotide polymorphisms in TL1A associated with risk for inflammatory bowel disease (IBD). ${ }^{17,18}$ TL1A is known to regulate adaptive immune responses in the gut and lung. Mice deficient for TL1A or DR3 (TNFRSF25; the only known receptor for TL1A) have reduced severity of diseases in autoimmune disease models such as asthma model, colitis model, or experimental autoimmune encephalomyelitis model, suggesting that this pathway is important in shaping various immune responses, including type 2 immune responses. ${ }^{16}$ TL1A has been ascribed a key role in mucosal immunity. ${ }^{16}$ Overexpression of TL1A results in small intestinal inflammation, suggesting their importance at mucosal barriers. ${ }^{19-21}$ DR3 is expressed on $\mathrm{CD}_{161}{ }^{+}$gut-homing T cells that respond to TL1A in vitro by expressing proinflammatory cytokines such as interferon- $\gamma$, TNF, IL6, and IL17. ${ }^{22}$ Moreover, recent data suggest that TL1A induces IL13 production also from non-T/ natural killer T-cell compartment in vivo, ${ }^{19}$ suggesting innate cells may also respond to TL1A.

Here we elucidate that TL1A is a novel player in ILC2 mucosal biology. We subjected ILC2 cells to microarray and report that DR3 is constitutively expressed on ILC2s. Engagement of DR3 with TL1A promoted ILC2 expansion, survival, and function in vitro and in vivo. We demonstrate that the TL1A/DR3 pathway plays a key role in promoting ILC2s at mucosal barriers and, using relevant receptor-deficient and Rag-deficient mice, we show this was specific to TL1A and independent of IL25 and IL33. Moreover, mice deficient for DR3 not only had impaired responses to control helminthic infections in the gut due to reduced functions of ILC2, but also failed to mount ILC2mediated type 2 inflammation in the lung after nasal challenge with papain, providing a functional relevance for this pathway in physiological inflammatory responses. Our data demonstrate a key role for TL1A in promoting ILC2 proliferation and activation at mucosal barriers.

\section{RESULTS}

\section{ILC2s constitutively express DR3}

IL25 and IL33 are two key cytokines that support ILC2 differentiation and function. ${ }^{23}$ Interestingly, although mice deficient for IL25 or IL33 or their receptors have impaired ILC2 responses, ${ }^{6,12-14}$ some functional ILC2s still remain, indicating the existence of additional pathways that support ILC2 biology. Consequently, we determined whether other pathways are necessary for eliciting ILC2 responses. We identified $d r 3$ as a highly expressed gene on human ILC2s ( $\mathrm{Lin}^{-} \mathrm{CRTH} 2^{+}$ $\mathrm{CD} 161^{+} \mathrm{CD} 127^{+}$cells) by microarray analysis and corroborated this by real-time quantitative PCR (Supplementary Figure S1a-d online). We confirmed cell-surface DR3 protein expression by fluorescence-activated cell sorting (FACS) on human ILC2s from human blood $\left(\mathrm{Lin}^{-} \mathrm{CRTH} 2{ }^{+} \mathrm{CD} 161^{+} \mathrm{CD} 127^{+}\right.$cells; (Figure 1a) and murine $\mathrm{ILC} 2 \mathrm{~s}\left(\mathrm{Lin}^{-} \mathrm{Scal}{ }^{+} \mathrm{CD} 127^{+} \mathrm{ST} 2^{+}\right.$ cells; Supplementary Figure S2a online) from mesenteric lymph node (mLN) and lung as well as in bone marrow $\left(\right.$ KLRG1 $^{-}$ILC2 precursors $^{24,25}$ ) from naive wild-type (WT) mice (Figure 1b).
We next determined a functional role for DR3 on ILC2s. Murine ILC2s were purified, expanded in vitro with ILC2 cytokine cocktail (IL2, IL7, IL25, and IL33), and their lineage confirmed by IL5 production in response to IL25 and IL33 (Supplementary Figure S2b online). FACS analysis confirmed that these cells retained surface expression of DR3 (Figure 1c). Importantly, stimulation of the ILC2s with recombinant TL1A (rTL1A) alone, in the absence of exogenous IL2 and IL7, significantly induced the production of IL5 and IL13 in vitro (Figure 1c).

\section{TL1A directly promotes ILC2s in vivo}

Given ILC2 responsiveness to TL1A, we reasoned that TL1Amediated immunopathology might be driven, in part, by innate lymphocytes, such as ILC2s. In order to further study the functions of TL1A/DR3 pathway on ILC2s, we generated transgenic mice expressing TL1A, under the CD11c promoter, and crossed the transgenic mice with $\mathrm{Rag}^{-/-}$mice (Supplementary Figure S3a,b online). Strikingly, transgenic expression of TL1A induced a significant increase in the levels of IL5 and IL13 in the ileum, even in the absence of adaptive immune cells (Supplementary Figure S3c online).

To further confirm these results, we directly administered

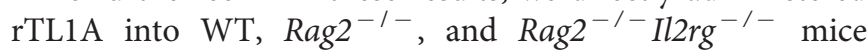
intraperitoneally (i.p.) to assess IL5 production in the peritoneum. Exogenous TL1A delivered i.p. elicited levels of IL5 in Rag2 ${ }^{-1-}$ mice quantitatively similar to that in WT mice, thereby excluding a contribution from $\mathrm{T}$ cells, whereas

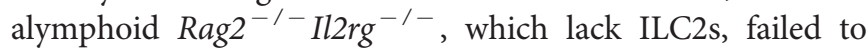
elicit IL5 production in response to rTL1A (Figure 2a). As expected, rTL1A failed to promote IL5 in $\mathrm{Dr}^{-/-}$(Figure 2b). In addition to increased IL5 production, rTL1A also increased the number of ILC2s in the mLN or lungs of WT and Rag2 ${ }^{-/-}$ mice after i.p. or intranasal challenge with rTL1A, respectively (Figure 2c,d). Together, these data demonstrate that TL1A is sufficient to induce ILC2s directly and independent of adaptive immune cells.

\section{TL1A/DR3 pathway is required for Nippostrongylus brasiliensis worm clearance}

Several studies have shown that ILC2s are a prominent source of IL5 and IL13 during a protective immune response to helminth infection. ${ }^{5,6,12} \mathrm{~N}$. brasiliensis is a helminthic worm infection that elicits a type 2 immune response, characterized by induction of ILC2s in the lung and the small intestine, which is necessary for worm clearance. ${ }^{26}$ We confirmed that DR3 is expressed on ILC2s-from mLN, lung, and bone marrow-in mice infected with $N$. brasiliensis (Figure 3a). Cohorts of WT and $D r 3^{-1-}$ mice were exposed to stage-3 larvae of $N$. brasiliensis and by day 10, WT mice had efficiently cleared all worms from their intestine, whereas the $\mathrm{Dr} 3^{-1-}$ mice had not (Figure $3 \mathbf{b}$ ). Analysis of infected mice on day 7 revealed significantly reduced numbers of ILC2s in the bronchoalveolar lavage fluid (BALF) of $\mathrm{Dr}^{-/-}$compared with WT mice, whereas the total $\mathrm{CD} 4^{+}$Th cells were unaffected (Figure 3c). In concordance with reduced ILC2 numbers, the lungs of infected $\mathrm{Dr}^{-/-}$mice had significantly less inflammation 
a Ex vivo human ILC2

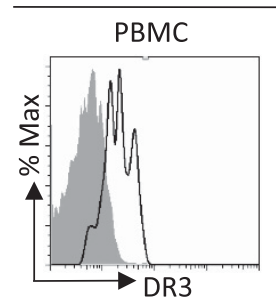

b

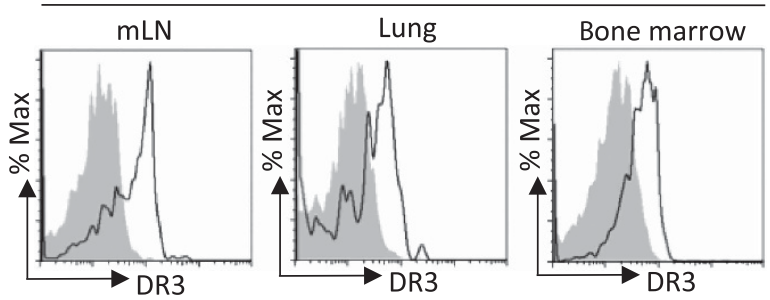

C In vitro murine ILC2
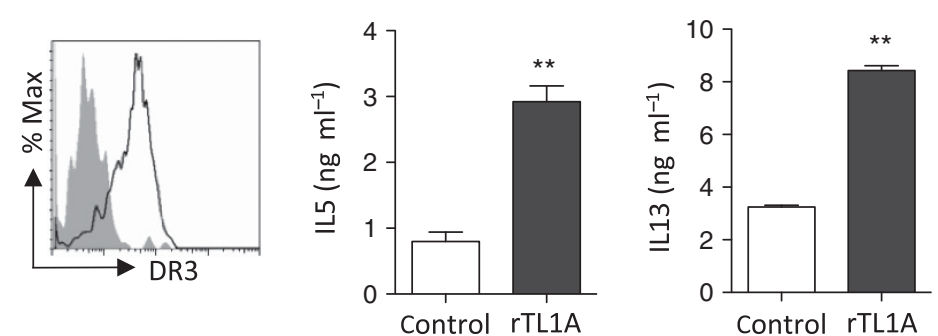

Figure 1 TL1A promotes innate type 2 lymphoid cell (ILC2) effector function in vitro. (a) Representative histogram of DR3 expression (black line histogram) ex vivo on human ILC2s ( Lin ${ }^{-}$CRTH2 ${ }^{+} \mathrm{CD} 161^{+} \mathrm{CD} 127^{+}$) isolated from peripheral blood mononuclear cells (PBMCs). Shaded histogram represents stain with control isotype. (b) Representative histogram of DR3 expression (black line histogram) ex vivo on murine ILC2s $\left(\mathrm{Lin}^{-} \mathrm{Sca} 1^{+} \mathrm{CD} 127^{+} \mathrm{ST} 2^{+}\right)$isolated from mesenteric lymph nodes $(\mathrm{mLNs})$, lung, or bone marrow of naive C57BL/6 mice. Shaded histogram represents stain with control isotype. (c) Representative histogram of DR3 expression (black line histogram) or isotype control (shaded histogram) on murine ILC2s after in vitro expansion; and interleukin 5 and 13 (IL5 and IL13) levels in culture supernatants with recombinant murine TL1A (rTL1A, black bars) or control (white bars) in the absence of exogenous IL2 and IL7. Data shown are representative of two independent experiments with triplicate wells in each one. Data are plotted as mean and s.d. ${ }^{\star \star} P<0.01$ by Student's $t$-test to corresponding control.
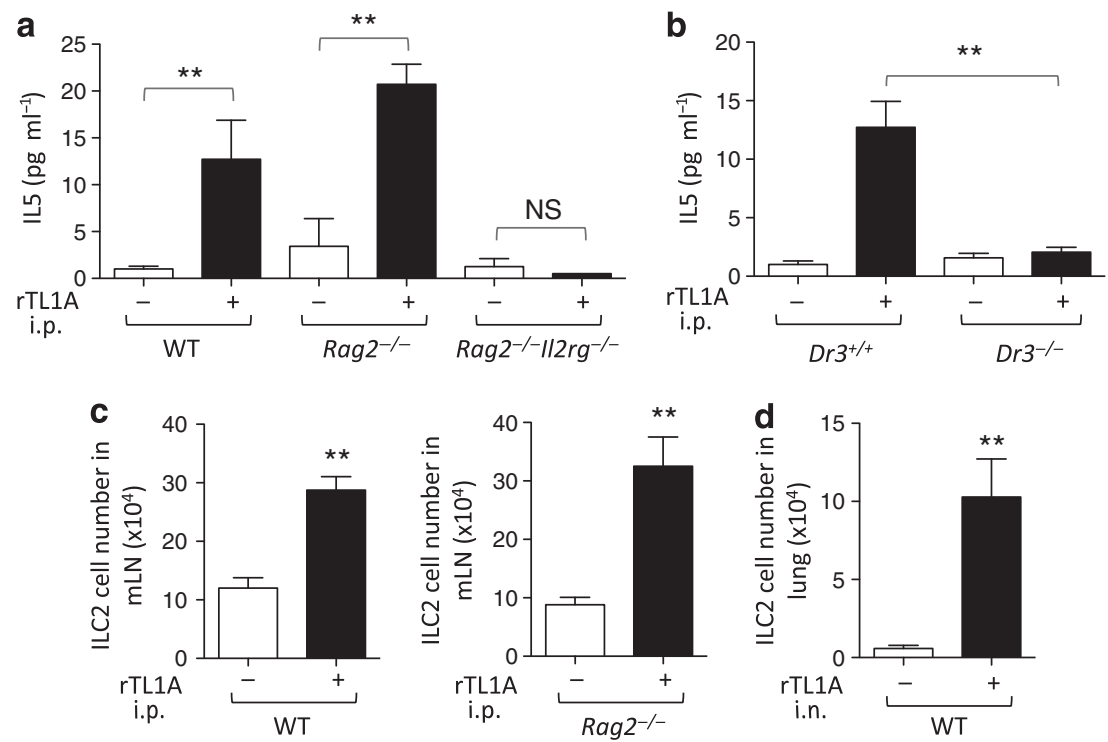

Figure 2 TL1A elicits innate type 2 lymphoid cells (ILC2s) in vivo. (a) Interleukin 5 (IL5) levels in peritoneal fluid from C57BL/6 (wild-type

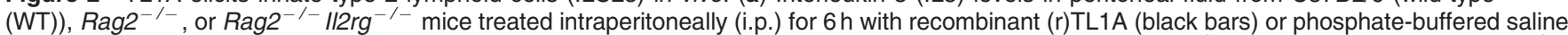
(PBS; white bars). Data are plotted as mean and s.e.m. ( $n=5$ per group). (b) IL5 levels in peritoneal fluid from Dr3 ${ }^{-/-}$mice or WT littermates (Dr3 ${ }^{+/+}$) treated i.p. for $6 \mathrm{~h}$ with rTL1A (black bars) or PBS (white bars). Data are plotted as mean and s.e.m. ( $n=5$ per group). (c) Enumeration of mesenteric lymph node (mLN) ILC2s from C57BL/6 (WT; left) or Rag2 ${ }^{-1-}$ (right) mice treated i.p. with rTL1A (black bars) or PBS (white bars) daily for 4 consecutive days ( $n=5$ per group). (d) Enumeration of ILC2s from whole-lung tissue of C57BL/6 (WT) mice exposed intranasally (i.n.) with rTL1A (black bars) or PBS (white bars) daily for 4 consecutive days ( $n=8$ per group). Data are representative of at least two independent experiments and plotted as mean and s.e.m. ${ }^{* \star} P<0.01$ by Student's $t$-test. NS, not significant.

(Supplementary Figure S4a,b online). Despite comparable Th cell numbers in the BALF, levels of the type 2 cytokines IL 5 and IL13 were all reduced in the BALF in $D r 3^{-/-}$mice upon infection, implying that TL1A/DR3 pathway may act to amplify the overall type 2 immune response in the infected lungs (Figure 3d). These data suggest that $D r 3^{-1-}$ mice were unable 
a
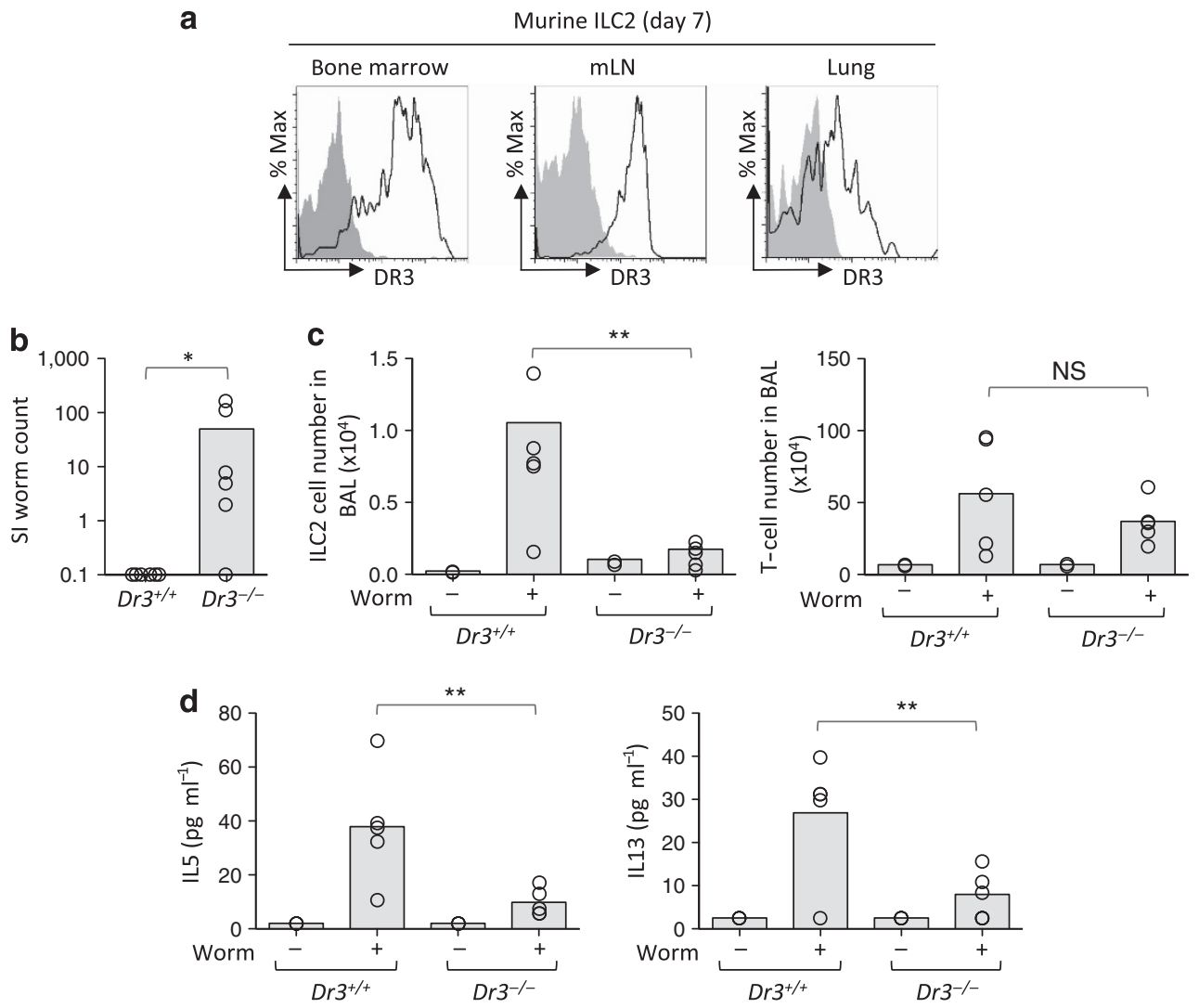

Figure 3 DR3-deficient mice have impaired innate type 2 lymphoid cell (ILC2) responses to helminth infection. (a) Representative histogram of DR3 expression (black line histogram) or isotype control (shaded histogram) on murine ILC2s from mesenteric lymph nodes (mLNs), lung, or bone marrow of Nippostrongylus Brasiliensis-infected C57BL/6 mice on day 7 after infection. (b) Total number of worms in small intestine (SI) of $N$. brasiliensisinfected $D r 3^{-/-}$mice or $D r 3^{+/+}$wild-type (WT) littermates at 10 days after infection. (c) Cell counts of ILC2s (left) and T cells (left) in the bronchoalveolar lavage fluid (BALF) of uninfected (white bars) or $N$. brasiliensis-infected (black bars) Dr3 ${ }^{-1-}$ mice or Dr3 ${ }^{+/+}$WT littermates. (d) Interleukin 5 and 13 (IL5 and IL13) levels in the BAL fluid (BALF) of uninfected (white bars) or N. brasiliensis-infected (black bars) Dr3 ${ }^{-1-}$ mice. Each dot in b-d represents data obtained from an individual animal. Data are representative of two independent experiments and the mean is plotted as bar graph $(n=3-6$ per group). ${ }^{*} P<0.05$ and ${ }^{* *} P<0.01$ by Student's $t$-test. NS, not significant.

to effectively clear the helminth worm infection because of the reduced numbers of ILC2s, although, as $\mathrm{CD} 4^{+} \mathrm{T}$ cells are still present in the $\mathrm{Dr} 3^{-/-}$mice, we cannot exclude an additional contribution of DR3-driven IL5 and IL13 from these adaptive immune cells to worm clearance. Together, this supports a functional role for DR3 in regulating ILC2s in vivo.

\section{TI1A/DR3 pathway is required for ILC2 responses in lung to papain challenge}

Studies have shown that intranasal administration of papain will induce robust lung inflammation that depends on ILC2s. ${ }^{27}$ Therefore, we used this model to evaluate whether DR3 is required for the generation of ILC2s in the lung. WT and $\mathrm{Dr}^{-/-}$mice were challenged with papain (intranasal) three times, and on day 7 immune cells were quantitated in BALF and lung tissue. ILC2s were easily enumerated in the BALF from WT mice, but were undetectable in $D r 3^{-1-}$ mice (Figure 4a). Eosinophils respond to IL5 and are commonly associated with mucosal immune responses. Eosinophils in the BALF after papain challenge were significantly reduced in the $\mathrm{Dr} 3^{-/-}$mice (Figure 4b). Although some ILC2s were still detected in the lung tissue in $\mathrm{Dr}^{-1-}$ mice, the numbers were significantly less than
WT (Figure 4c,d). In contrast, the total T-cell number and natural killer $\mathrm{T}$ cell number in lung tissues remain similar in WT and $D r 3^{-/-}$mice (Supplementary Figure S5a-c). To exclude the possibility that the reduced ILC2 numbers in $D r 3^{-/-}$mice, after papain challenge, were simply a reflection of lower CD127 levels used to enumerate these cells, we performed similar assessment in $\mathrm{Dr} 3^{-1-}$ mice, lacking adaptive immune cells, using additional ILC2 FACS markers. Dr3 ${ }^{-1-}$ mice were crossed to $\mathrm{Rag}^{-1-}\left(\mathrm{Rag}^{-/-} \mathrm{Dr} 3^{-/-}\right)$and subjected to intranasal papain challenge together with $R a g 2^{-/-}$control mice. ILC2s, identified as $\mathrm{Lin}^{-} \mathrm{sca}^{+}{ }^{+} \mathrm{ST} 2{ }^{+} \mathrm{CD} 278^{+}$, and eosinophils were enumerated in the lung tissue and BALF, respectively. As seen above, ILC2s in $R a g 2^{-/-} \mathrm{Dr} 3^{-/-}$were also significantly reduced after papain challenge compared with $\operatorname{Rag}^{-/-}$mice carrying WT Dr3 alleles (Figure 4e,f), as were eosinophils in the BALF (Figure 4g). These results suggest that DR3 is required for ILC2 survival/expansion in the lung to papain challenge.

\section{TL1A synergizes with IL25 and IL33 to enhance ILC2 effector function}

As our data support a role for TL1A on ILC2 expansion and function, we asked whether TL1A worked independently or in 

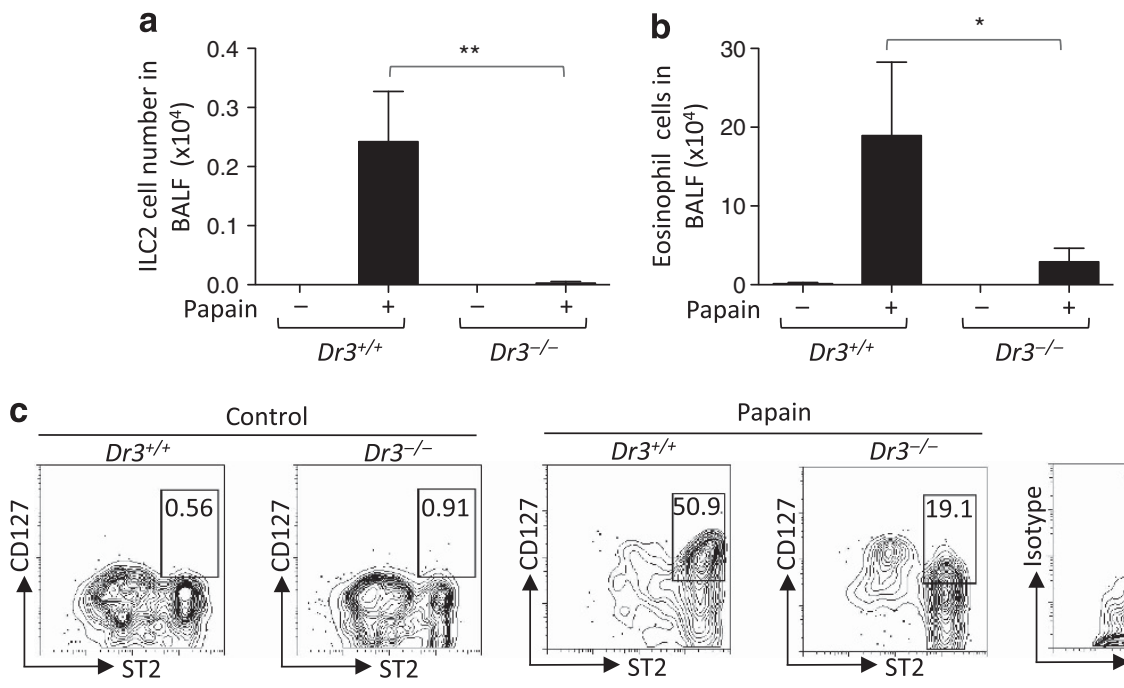

Papain
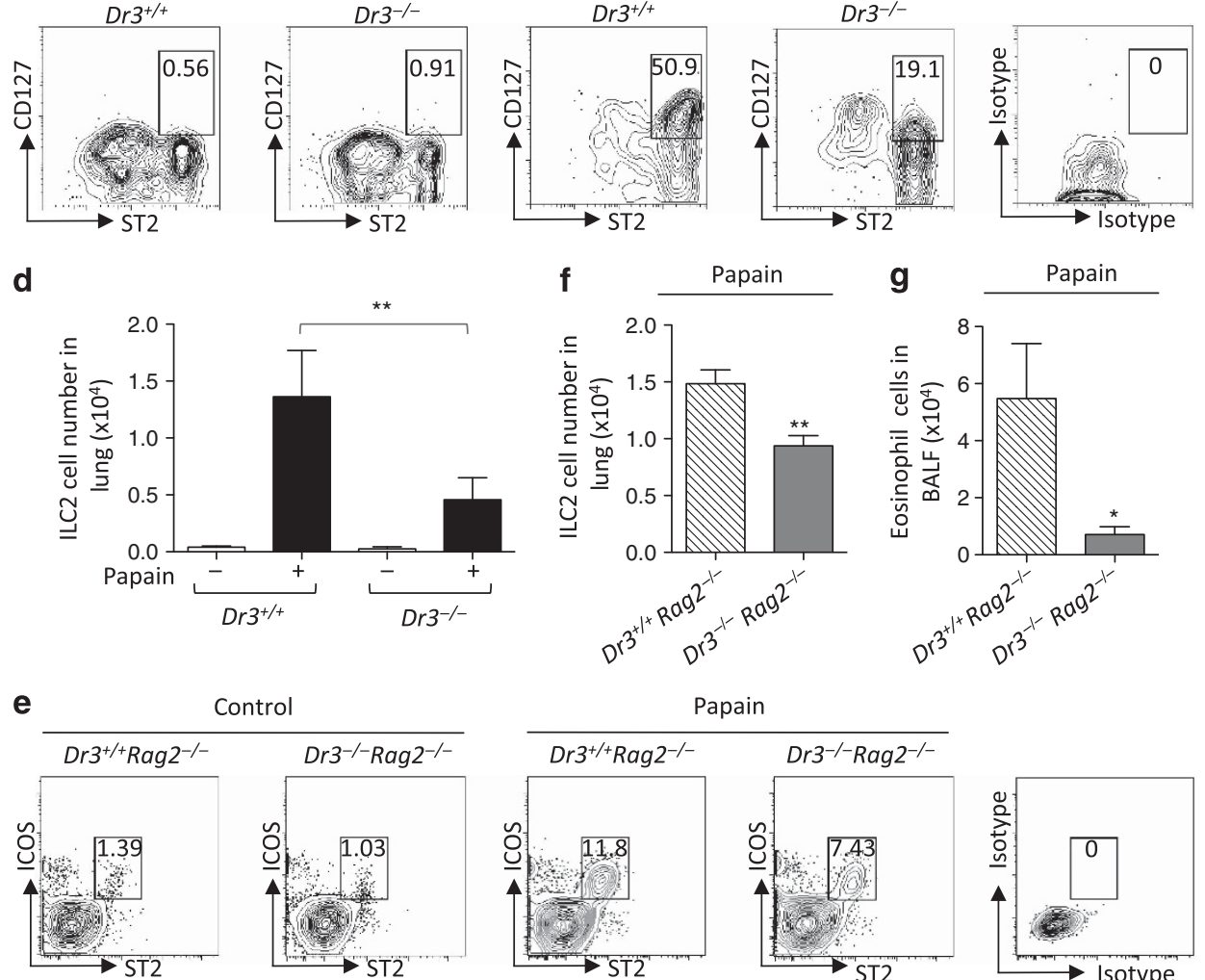

Control
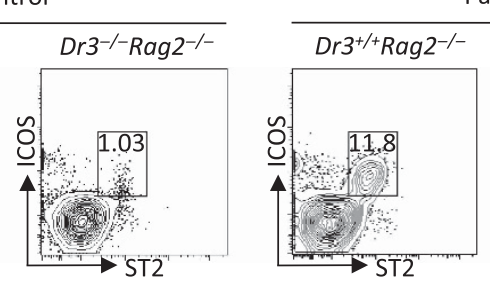

Papain
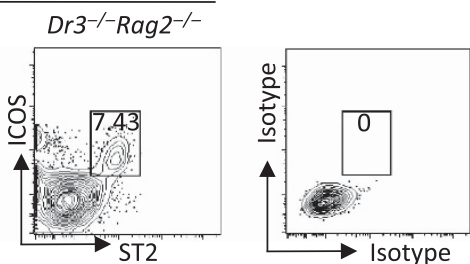

Figure 4 DR3-deficient mice have impaired innate type 2 lymphoid cell (ILC2) responses to papain challenge. (a) Enumeration of ILC2s from the bronchoalveolar lavage fluid (BALF) of $D r 3^{-/-}$mice or $D r 3^{+/+}$wild-type (WT) littermates treated with papain (black bars) or phosphate-buffered saline (PBS; white bars). (b) Quantification of eosinophils $\left(\mathrm{SSC}^{\text {hi }}\right.$ Siglec- $\mathrm{F}^{+} \mathrm{F} 4 / 80^{\text {int }}$ ) in BALF from Dr3 ${ }^{-/-}$mice or Dr3 ${ }^{+/+}$WT littermates treated with papain (black bars) or PBS (white bars). (c) Representative fluorescence-activated cell sorting (FACS) plots of Lin ${ }^{-} \mathrm{Sca}^{+}{ }^{+}$cells from whole-lung tissue of $D r 3^{-/-}$mice or $\mathrm{Dr} 3^{+/+}$WT littermates treated with papain or PBS. The ILC2s are gated as $\mathrm{Lin}^{-} \mathrm{Sca} 1^{+} \mathrm{ST} 2^{+} \mathrm{CD} 127^{+}$cells. The numbers in the plots show the percentages of cells in the indicated gates. (d) Enumeration of ILC2s from whole-lung tissue of $D r 3^{-/-}$mice or Dr3 $3^{+/+} \mathrm{WT}$ littermates treated with papain (black bars) or PBS (white bars) as shown in c. (e) Representative FACS plots of Lin ${ }^{-}$Sca1 ${ }^{+}$cells from whole-lung tissue of $\mathrm{Dr}^{-1-} \mathrm{Rag}^{-1-}$ mice or $\mathrm{Dr} 3^{+}{ }^{+} \mathrm{Rag}^{-{ }^{-\prime}}$ control littermates treated with papain or PBS as control. The ILC2s are gated as $\mathrm{Lin}^{-} \mathrm{Sca} 1^{+} \mathrm{ST} 2^{+} \mathrm{CD} 278^{+}$ cells. The numbers in the plots show the percentages of cells in the indicated gates. (f) Enumeration of ILC2s from whole-lung tissue of $D r 3^{-1-}$ Rag2 ${ }^{-1-}$ mice (gray) or $\mathrm{Dr} 3^{+/+} \mathrm{Rag}^{-/-}$littermates (stripped) treated with papain as shown in e. (g) Quantification of eosinophils in BALF from Dr3 ${ }^{-/-}$Rag2 $^{-/-}$ mice (gray) or $D r 3^{+/+} \operatorname{Rag}^{-/-}$littermates (stripped) treated with papain. Data in $\mathbf{a}, \mathbf{b}, \mathbf{d}, \mathbf{f}$, and $\mathbf{g}$ are plotted as mean and s.e.m. ( $n=5$ per group). Data are representative of two independent experiments. ${ }^{\star} P<0.05$ and ${ }^{\star \star} P<0.01$ by Student's $t$-test.

synergy with IL25 or IL33 pathways. Effects of exogenous TL1A, administered i.p, on cytokine production were compared in WT mice or mice deficient for DR3 $\left(D r 3^{-/-}\right)$, IL25R $\left(I l 17 \mathrm{rb}^{-/-}\right)$, or IL33R $\left(S T 2^{-/-}\right)$. TL1A induced similar levels of IL5 in peritoneal fluid in WT, Ill $7 \mathrm{rb}^{-1-}$, and $S T 2^{-1-}$ mice (Figure 5a,b), indicating that TL1A was sufficient to induce and activate ILC2s independent of IL25 or IL33 signaling. Next, we asked whether TL1A/DR3 pathway was required for ILC2 development and mediating the downstream functions of IL33 and IL25. Basal ILC2 numbers were comparable in WT and
$\mathrm{Dr}^{-/-}$mice, implying DR3 was not required for ILC2 development. Moreover, the surface levels of the markers ST2 and CD127 used for enumeration of ILC2 were similar in WT and $\mathrm{Dr} 3^{-1-}$ mice at baseline and remain positive after recombinant protein challenge. DR3 was not essential for ILC2 induction, as exogenous recombinant IL33 or IL25 were capable of inducing ILC2 in $\mathrm{Dr} 3^{-/-}$mice (Figure 5c). Interestingly, ILC2 numbers induced by IL33 were similar in WT and Dr3 ${ }^{-/-}$mice; however, ILC2 induced by IL25 were significantly reduced in the $\mathrm{Dr}^{-/-}$mice (Figure 5c). 
a

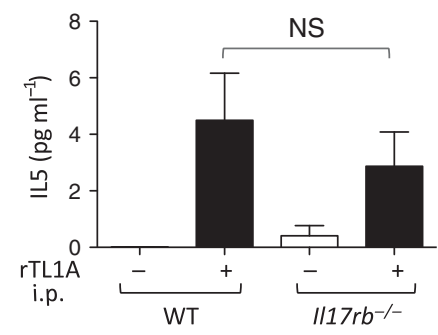

b

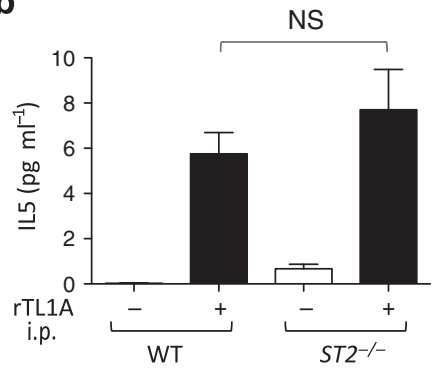

C

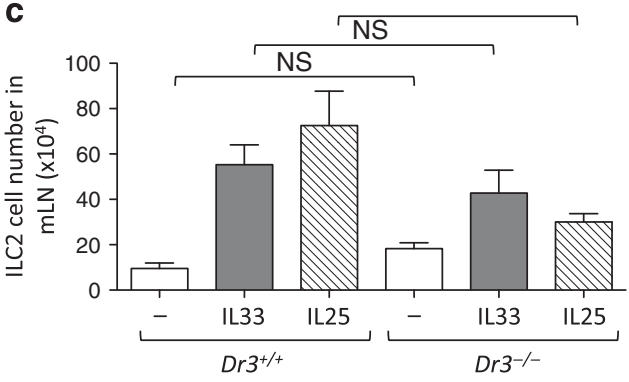

d

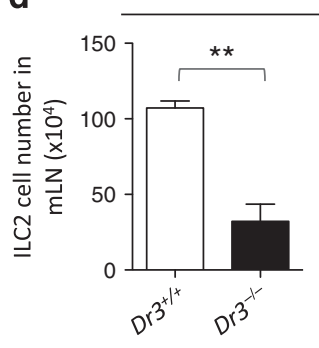

IL25

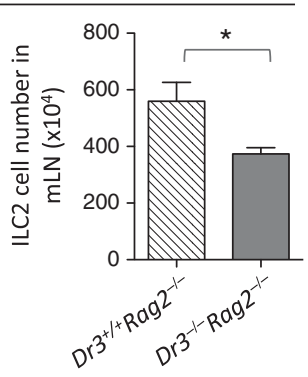

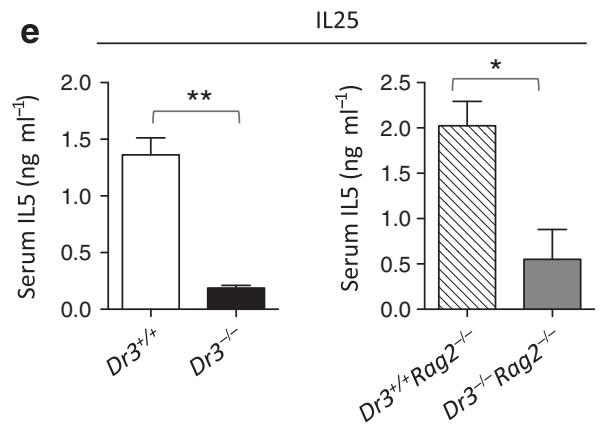

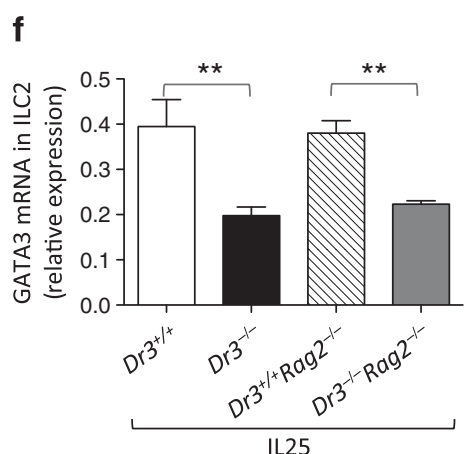

g

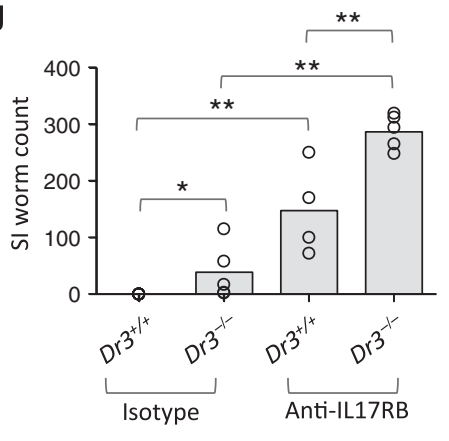

Figure 5 TL1A is sufficient to activate innate type 2 lymphoid cells (ILC2s) independent of interleukins 25 and 33 (IL25 and IL33). (a, b) IL5 levels in peritoneal fluid from (a) $/ / 17 \mathrm{rb}^{-/-}$or (b) ST2 ${ }^{-/-}$mice or corresponding wild-type controls (WT) treated intraperitoneally (i.p.) with recombinant (r)TL1A (black bars) or treated with phosphate-buffered saline (PBS) (white bars) for $6 \mathrm{~h}$ ( $n=5$ per group). (c) Enumeration of ILC2s from mesenteric lymph nodes (mLNs) of WT littermates $\left(D r 3^{+/+}\right.$) or Dr3 ${ }^{-/-}$mice treated i.p. with IL33 (gray), IL25 (striped), or PBS (white) daily for 4 consecutive days ( $n=5$ per group). (d, e) Enumeration of (d) mLN ILC2s and (e) serum IL5 levels in Dr3 ${ }^{-1-}$ mice (black bars) or littermates (Dr3 ${ }^{+/+}$, white bars), and $D r 3^{-/-}$Rag2 $2^{-/-}$mice (gray bars) or littermates (Dr3 ${ }^{+/+} \operatorname{Rag}^{-/-}$, striped bars) treated with IL25 daily for 4 consecutive days $(n=4-5$ per group). (f) GATA3 mRNA expression in sorted mLN ILC2s from IL25-treated mice in d (Dr3 ${ }^{-/-}$, black bars; $D r 3^{+/+}$littermates, white bars; and Dr3 ${ }^{-1-}$ Rag2 $2^{-1-}$, gray bars; and $D r 3^{+/+} \operatorname{Rag}^{-/-}$, striped bars; $n=4-5$ per group). Data in a-f are plotted as mean and s.e.m. (g) Total number of worms in small intestine of $N$. brasiliensis-infected $D r 3^{-/-}$mice or $D r 3^{+/+}$WT littermates treated with anti-IL17RB blocking antibody or isotype control, 10 days post infection. Each dot represents data obtained from an individual animal. Mean is plotted as bar graph $\left(n=5\right.$ per group). ${ }^{\star} P<0.05$ and ${ }^{\star \star} P<0.01$ by Student's $t$-test. NS, not significant; SI, small intestine.

To exclude the indirect effects of IL25 and/or TL1A on adaptive immune cells, we tested the synergy of IL25 and TL1A in promoting ILC2s in Rag-deficient mice. Our data showed that, as seen in Rag-sufficient $D r 3^{-/-}$mice, IL25 induced less ILC2s in Rag $2^{-/-}$deficient for DR3, likely accounting for the significant reduction of IL5 in these mice (Figure 5d,e). Recently, it has been shown that GATA3 is critical for ILC2 differentiation and maintenance. ${ }^{24,25}$ GATA3 expression upon IL25 stimulation was significantly decreased in ILC2s that lack DR3 (Figure 5f). Consequently, expression of ILC2-associated genes downstream of GATA3, in ILC2s sorted from IL25-treated $\mathrm{Dr}^{-1-}$ mice, were also inhibited, including IL13, amphiregulin, and ST2 (Supplementary Figure S6a-c). IL17RB mRNA in ILC2s in response to IL25 was, however, unaltered by DR3 deficiency (Supplementary Figure S6d). Although we observed a predominant synergy of TL1A with IL25 rather than with IL33 in ILC2s in vivo, we did observe that both IL25 and IL33 synergized with TL1A in in vitro expanded murine ILC2s (Supplementary Figure S6e,f). To confirm whether TL1A synergized with IL25 in vivo, we repeated the $N$. brasiliensis infection in $\mathrm{Dr}^{-/-}$as above, but with the addition of antiIL17RB (an IL25 receptor blocking antibody; Figure 5g). As before, the lack of DR3 resulted in delayed worm clearance, as enumerated from the small intestines. Blocking of IL25 in WT mice also significantly delayed worm clearance as previously reported. ${ }^{6,7}$ The addition of anti-IL17RB to DR3-deficient mice resulted in significantly impaired clearance of the parasite and higher worm burden than in mice inhibited for DR3 or IL25 
a

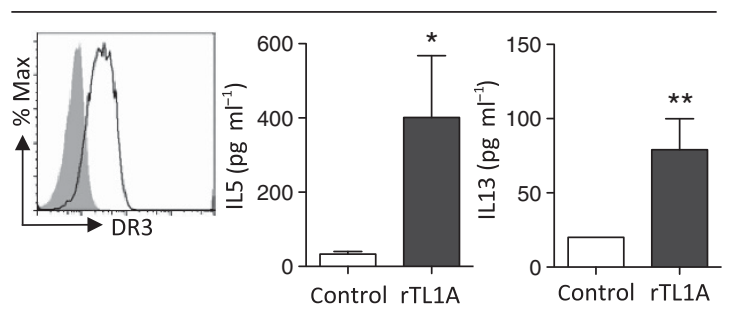

b

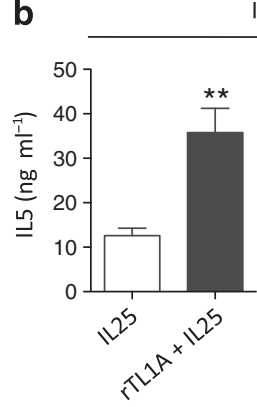

IL2 + IL7

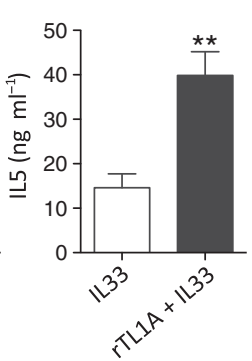

C
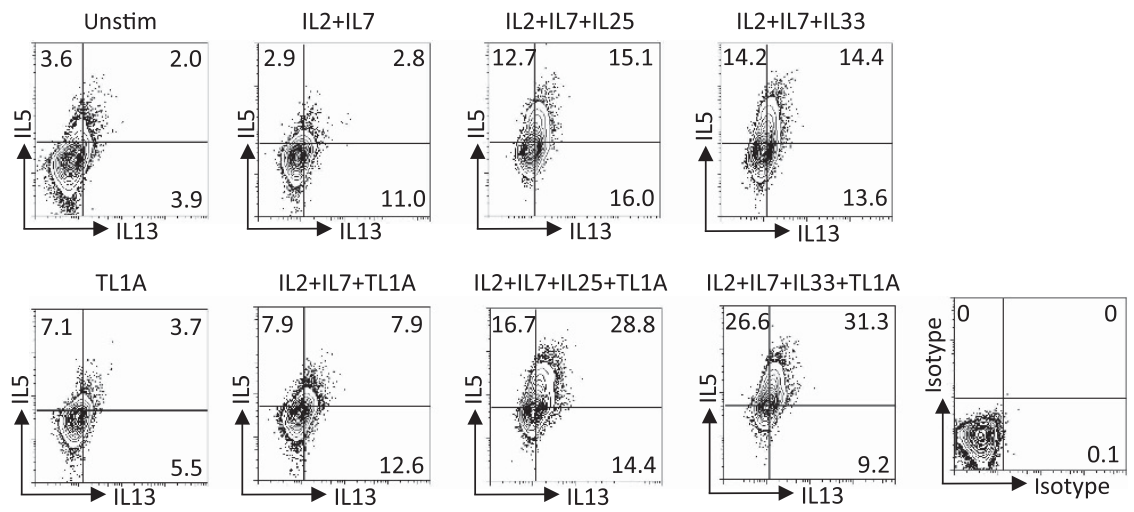

d
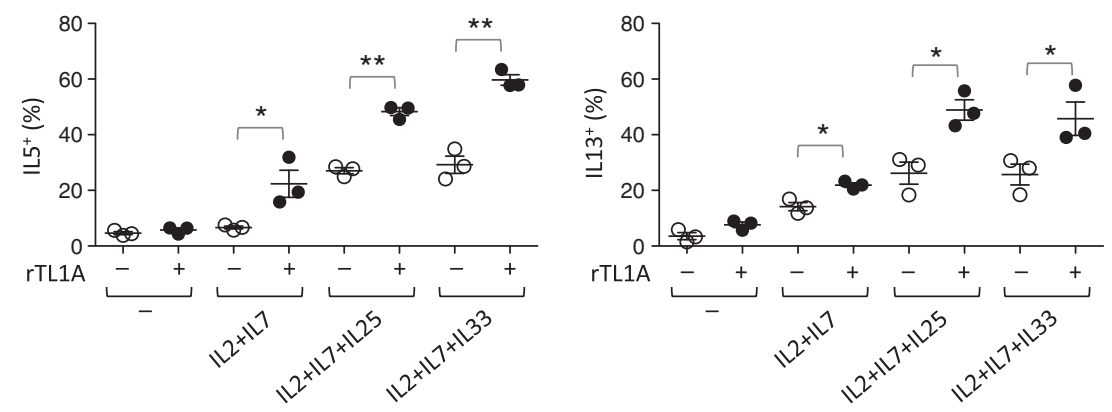

Figure 6 TL1A promotes human innate type 2 lymphoid cell (ILC2) function in synergy with interleukins 25 and 33 (IL25 and IL33). (a) Representative histogram of DR3 expression (black line histogram) or isotype control (shaded histogram) on human ILC2s after in vitro expansion; and IL5 and IL13 levels in culture supernatants with recombinant human TL1A (rTL1A, black bars) or control (white bars) in the absence of exogenous IL2 and IL7. Data shown are representative of two independent experiments with three donors each. (b) IL5 responses of in vitro expanded human ILC2s to IL25 or IL33 alone (white bars) or rTL1A plus IL25 or IL33 (black bars) in the presence of suboptimal IL2 and IL7. (c, d) Representative fluorescence-activated cell sorting (FACS) plots (c) and quantification (d) of the percentage of in vitro generated human ILC2s producing IL5 and IL13. Each dot represents data obtained from an individual donor. The mean and s.e.m. is also plotted (lines). Unstim, unstimulated. ${ }^{\star} P<0.05$ and ${ }^{\star \star} P<0.01$ by Student's $t$-test.

pathways alone. These data show that TL1A can synergize with IL25, and maybe IL33 to influence ILC2 generation and effector function, and suggests that TL1A may play feedforward role on ILC2s at different locations.

\section{Human ILC2s}

To extend the observation that TL1A acts alone and in synergy with IL25 and IL33, we performed similar experiments with in vitro expanded human ILC2s (Supplementary Figure S1a,d online). FACS analysis confirmed that human ILC2s retained surface expression of DR3 after expansion with ILC2 cytokine cocktail (Figure 6a). As seen with murine ILC2s, stimulation of human ILC2s with rTL1A alone, in the absence of exogenous IL2 and IL7, also significantly induced the production of IL5 and IL13 in vitro (Figure 6a). Importantly, rTL1A and IL25 or
IL33 together elicit significantly higher IL5 production from ILC2s than IL25 or IL33 alone (in the presence of suboptimal IL2 plus IL7; Figure 6b). This is further confirmed by intracellular FACS analysis (Figure $\mathbf{6 c}$,d). These observations indicate that TL1A can regulate human ILC2s and function synergistically with the two key ILC2 regulators IL25 and IL33 to enhance human ILC2 effector functions.

\section{DISCUSSION}

Here we elucidate a novel player in ILC2 mucosal biology, the TNFSF member TL1A. The expression of TL1A in lung and gut inflammation and its genetic association with IBD in genomewide association studies place it in a key role in regulating mucosal immune response. Our data provide the first link of the TNFSF with innate lymphoid cells activation and function, and 
suggest a link of TL1A with primordial immune responses. Other TNFSF members are dysregulated during mucosal inflammation. Indeed, blockade of TNF affords therapeutic benefit in some IBD patients; however, TNF does not modulate ILC2 function (Supplementary Figure S7 online). Our data show that TL1A can synergize with IL25, and to a lesser extent IL33. TSLP was recently identified to play a synergistic role with IL33 in ILC2s in the skin of atopic dermatitis patients. ${ }^{15}$ These data suggest that tissue-specific cytokines may work together with IL25 and IL33 in a feed forward manner to reinforce type 2 immune response.

All ILCs lack lineage markers ( $\mathrm{Lin}^{-}$) but express the lymphoid progenitor marker IL7R $\alpha$ (CD127) and the cytokine common gamma $(\gamma c)$ receptor chain, and require IL7 for development. ${ }^{10}$ ILC2s additionally express the receptor for IL33, T1/ST2, and the receptor for IL25, IL17RB, ${ }^{2}$ and these cytokines are important drivers of ILC2 in the gut and lung. ${ }^{6,9}$ Here we used these markers to identify mouse ILC2s from BALF, lung, and mLN and found that DR3 is constitutively expressed by ILC2s at these tissues. DR3 was also expressed by immature ILC2s $\left(\mathrm{KLRG1}^{-}\right.$) in mouse bone marrow. However, DR3-deficient mice had similar numbers of ILC2s as WT littermates, suggesting that DR3 is not essential for ILC2 development. Nevertheless, DR3 is important for ILC2 activation and effector function. We show that the ligand for DR3, TL1A, could elicit ILC2-derived effector cytokines in vitro and in vivo in WT or Rag2-deficient mice. Although the overall magnitude of TL1 A effects on IL5 and IL13 was less than IL25 or IL33, the TL1A/DR3 pathway was essential for generation of ILC2s in vivo, as DR3-deficient mice not only had impaired responses to control helminthic infections in the gut, but also failed to mount ILC2-mediated type 2 inflammation in the lung after nasal challenge with papain. Moreover, Rag-deficient $\mathrm{Dr} 3^{-/-}$mice that lack adaptive immune cells were also defective in ILC2 induction upon papain challenge, further supporting a direct functional role for DR3 on ILC2 generation and function. Notably in these models, DR3 deficiency did not alter the number of $\mathrm{CD}^{+}{ }^{+} \mathrm{T}$ cells or natural killer $\mathrm{T}$ cells, suggesting that DR3 was required specifically for the generation of pathogenic ILC2s only.

Despite the dependence of ILC2 on IL25 and IL33 for activation and competent effector function, mice deficient in either of these signaling pathways retained some functional ILC2s. ${ }^{12-14}$ TL1A was capable of inducing ILC2s and IL5 in $I l 17 \mathrm{rb}^{-/-}$or ST2 ${ }^{-/-}$mice. Furthermore, we found that TL1A can significantly synergize with IL25 in vivo. ILC2s are detectable in $\mathrm{Dr} 3^{-1-}$ mice, and IL33 or IL25 were capable of expanding these cells in vivo. Interestingly, ILC2 numbers induced by IL33 were similar in WT and $\mathrm{Dr}^{-/-}$mice; however, ILC2 induced by IL25 were significantly reduced in the $D r 3^{-/-}$mice. Although we observed a predominant synergy of TL1A with IL25 rather than with IL33 in ILC2s in vivo, we did observe that both IL25 and IL33 synergized with TL1A in in vitro expanded murine and human ILC2s. Notably, we show that mice in which both DR3 and IL25 were inhibited were unable to clear helminth worm infections, and worm burdens were significantly higher on day 10 after infection compared with inhibition of DR3 or IL25 alone. This may be because of the fact that exogenous recombinant IL33 is a potent inducer of other cytokines, including IL25 as well as TSLP, ${ }^{28}$ that may be sufficient to elaborate a maximal ILC2 response, in the absence of DR3. Collectively, the data suggest that TL1A can synergize with IL25 and IL33, and possibly other cytokines, to influence ILC2 generation and effector function.

The expression of TL1A at mucosal sites places it in a key position to regulate the magnitude of the innate response in a feedforward manner. Indeed, other TNFSF members not only regulate adaptive immune cells, but also play essential roles in regulating innate immune responses, providing a link and interplay between cells in these different immune responses. LTi cells are innate lymphoid tissue inducer cells and, as the name implies, induce lymphoid tissue and are therefore cells that can regulate early inflammatory responses, especially in mucosal tissue. TNF, LT, OX40, and LIGHT are all known to regulate LTi cells in the gut. ${ }^{29}$ Interestingly, TL1A has been shown to be able to regulate OX40 expression on LTi cells in vitro, implicating a broader effect of TL1A on innate immune cells. ${ }^{30}$ Unlike these other TNFSF members, TL1A also plays an important role in regulating ILC2s. Thus, TL1A may have a unique role among TNFSF members in mediating the crosstalk of innate and adaptive immune cells during type 2 responses.

Genome-wide association studies have identified singlenucleotide polymorphisms in TL1A associated with the risk for IBD. ${ }^{17,18}$ Intriguingly, transgenic overexpression of TL1A results in intestinal inflammation, specifically ileitis, associated with elevated IL13. ${ }^{19-21}$ Studies with DR3- or TL1A-deficient mice also highlight an essential role of the DR3/TL1A pathway in amplifying adaptive immune responses in various chronic inflammatory diseases such as in asthma and IBD. ${ }^{19,20,31,32}$ DR3 is not essential for Th cell activation or polarization of Th cells to effector Th1, Th2, and Th17 cells, but DR3 is expressed on all these Th subsets and synergizes with other signaling pathways to elaborate effector cytokines from these cells. ${ }^{33}$ Intriguingly, pathogenic gut-homing $\mathrm{T}$ cells, defined by the surface marker $\mathrm{CD} 161^{+}$, have been recently shown to express DR3, and to respond to TL1A but not TNF. ${ }^{22}$

Although a direct role for ILC2s in IBD pathogenesis is undocumented, these cells and type 2 effector cytokines, such as IL5 and IL13, can contribute to goblet cell proliferation and fibrosis. As such, activated ILC2s may underscore pathogenesis in Crohn's disease in which the ileum is affected. Interestingly, transgenic overexpression of TL1A mice result in ileitis. ${ }^{19-21}$ In our hands, the ileum of transgenic mice overexpressing TL1A expressed elevated IL5 and IL13, even when crossed onto a Rag2-deficient background. Moreover, $D r 3^{-/-}$mice failed to fully expulse $N$. brasiliensis worms, a helminth infection model that provokes a potent type 2 response and in which worm clearance is dependent on ILC2s in the gut. ${ }^{6}$

Our findings demonstrate a key role for TL1A in promoting ILC2 proliferation and activation at mucosal barriers and reveal a previous uncharacterized function for this TNFSF member in regulating primordial ILC biology. We show that TL1A alone is 
sufficient to activate ILC2s, but that it can also synergize with other inflammatory mediators in local mucosal and epithelial tissue environments. A parallel role for TSLP has been identified for ILC2s in the skin of atopic dermatitis patients. ${ }^{15}$ The significant role of TL1A in activating both adaptive immune cells and ILC2 implicate the TL1A/DR3 axis in the pathogenesis of IBD and asthma, and blockade of this pathway may afford clinical benefit in these diseases.

\section{METHODS}

Mice. TL1A transgenic mice were made using mouse TL1A complementary DNA (accession: NM_177371.3) driven by murine CD11c promoter. ${ }^{34}$ The 750 bp complementary DNA fragment encoding fulllength mouse TL1A was amplified by PCR to the $3^{\prime}$ of the $5.5 \mathrm{~kb}$ CD $11 \mathrm{c}$ promoter. The construct was linearized by ApoI digestion and purified by gel electrophoresis for microinjection. Founder mice were identified by PCR using primers specific for the human growth hormone sequence in the construct and bred to $\mathrm{C} 57 \mathrm{BL} / 6$ and $\mathrm{Rag} 2^{-/-}$mice. The TL1A mRNA expression was confirmed by RT-PCR with TL1Aspecific primers (Mm00770031_m1, Life Technologies, Carlsbad, CA).

$\mathrm{Dr}^{-{ }^{-}}$mice and $\mathrm{Il17} \mathrm{rb}^{-T_{-}}$mice were obtained from Lexicon Pharmaceuticals (The Woodlands, TX) ${ }^{35}$ and backcrossed onto $\mathrm{C} 57 \mathrm{BL} / 6$ or $\mathrm{BALB} / \mathrm{c}$ background, respectively. ST2 $2^{-1-}$ mice were kindly provided by Andrew McKinsey at the Medical Research

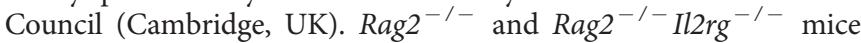
were from Taconic (Cambridge City, IN). TNFR-deficient mice lacking both TNFR1 (TNFRSF1a) and TNFR2 (TNFRSF1b) (Tnfrsf $1 a^{-/-} 1 b^{-/-}$), and wild-type B6129SF2/J, and BALB/c and C57BL/6 mice were all purchased from The Jackson Laboratory (Bar Harbor, ME). All animals used in this study were housed and maintained in pathogen-free conditions at Genentech (South San Francisco, CA) in accordance with the American Association of Laboratory Animal Care guidelines. All experimental studies were conducted under the approval of the Institutional Animal Care and Use Committees of Genentech Lab Animal Research.

Reagents. The extracellular domain portion of murine TL1A (amino acids (a.a.) 72-270) was cloned onto pST239 vector. The protein was purified from Escherichia coli on NiNTA column. The formation of trimer was confirmed by size-exclusion chromatography/multiangle light analysis. For recombinant murine IL33, the IL33 cytokine domain (a.a. 109-266) was cloned into a pET28 E. coli expression vector containing the $\mathrm{N}$-terminal $6 \times$ His-tag. For recombinant human IL33, the cytokine domain (a.a. 112-270) was cloned into the pET28 E. coli expression vector containing the $\mathrm{N}$-terminal $6 \times$ His-tag. For recombinant murine IL25, the murine IL25 cytokine domain (a.a. 17-169) was subcloned into a pRK expression vector containing the C-terminal $8 \times$ His-tag. For recombinant human IL25, the cytokine domain (a.a. 1-177) was cloned into pRK vector with the C-terminal FLAG tag and also produced in Chinese hamster ovary $(\mathrm{CHO})$ cells. The E. coli- and $\mathrm{CHO}$ cell-produced protein was purified under native condition over a NiNTA column followed by purification over a gel filtration column. Recombinant human IL2 is from Peprotech (Rocky Hill, NJ). Other recombinant cytokines used for cell culture are all from R\&D Systems (Minneapolis, MN).

Generation of anti-IL17RB blocking antibody. Monoclonal mouse anti-mouse IL17RB antibody was generated by immunizing IL17RBdeficient mice with fusion protein composed of mouse IL17RB extracellular domain (a.a. 18-282) and mouse IgG2a Fc domain. Clone $1 \mathrm{E} 8$ was selected based on its ability to block the interaction between IL17RB-Fc and plate-coated mouse IL25 and its ability to block IL25induced ILC2 expansion and IL5 production in vivo. The selected antiIL17RB antibody was then sub-cloned into vector of mouse IgG2a backbone with 2 mutations (D265A/N297A) known to abolish FcR binding and expressed in $\mathrm{CHO}$ cells for purification.

Flow cytometry. Single-cell suspensions from mLNs), bone marrow, isolated lung lymphocytes, or BALF were washed in phosphatebuffered saline (PBS) containing $5 \mathrm{~mm}$ EDTA and $0.5 \%$ bovine serum albumin. Cells were incubated for $15 \mathrm{~min}$ with $\mathrm{Fc}$ receptor block (clone 2.4G2, BD Pharmingen, San Diego, CA) before antibody staining. Propidium iodide was used to discriminate between viable and dead cells. All the antibodies and isotype-matched controls, unless otherwise addressed, were from BD Biosciences (San Diego, CA), eBiosciences (San Diego, CA), or Biolegend (San Diego, CA). Cells were stained with indicated antibodies and sorted on a BD FACS Aria or analyzed on a FACSCalibur or LSRII (BD Biosciences). Flow cytometry data were analyzed using FlowJo software (Treestar, Ashland, OR). For determination of absolute cell numbers, CaliBRITE APC Beads (BD Biosciences) were added before analyzing samples by flow cytometry, and total cell numbers were determined according to the manufacturer's instructions.

ILC2 isolation and in vitro expansion. Human peripheral blood mononuclear cells from healthy volunteers were obtained after informed consent and isolated by centrifugation over Ficoll-Paque Plus (Amersham Biosciences, Piscataway, NJ). Ethical approval for use of this material was obtained from the Western Institutional Review Board. Human ILC2s were sorted as $\mathrm{Lin}^{-} \mathrm{CRTH} 2{ }^{+} \mathrm{CD}^{-} 61^{+}$cells from peripheral blood $^{36}$ using the following antibodies: fluorescein isothiocyanate (FITC)-conjugated lineage markers for human lymphoid cells including anti-CD1a (HI149); anti-CD3 (OKT3); anti-CD4 (RPA-T4); anti-CD11c (3.9); anti-CD14 (MфP9); anti-CD16 (3G8); anti-CD19 (1D3); anti-CD34 (581); anti-CD56 (NCAM16.2); antiCD94 (DX22); anti-CD123 (6H6); anti-TCR $\alpha \beta$ (WT31); anti-TCR $\gamma \delta$ (B1); anti-BDCA2 (AC144; Miltenyi Biotec, Auburn, CA); and antiFceR1 (AER-37(CRA1)), Alexa 647-conjugated anti-human CRTH2 (BM16), phycoerythrin (PE)-conjugated anti-human CD161 (HP3G10), and PE-Cy7-conjugated anti-human CD127 (R34.34; Beckman Coulter, Brea, CA). Sorted cells were expanded in vitro in Yessel's media with $1 \%$ human $\mathrm{AB}$ serum (Sigma Aldrich, St Louis, MO) containing rhIL2, rhIL7, rhIL33, and rhIL25 (each at $50 \mathrm{ng} \mathrm{ml}^{-1}$ ) for 7-10 days. Cells were rested in minimal cytokines (rhIL2 and rhIL7 only, each at $10 \mathrm{ng} \mathrm{ml}^{-1}$ ) for 2 days before they were used for experiments. For restimulation, suboptimal levels of cytokines were used (rhIL2 at $10 \mathrm{ng} \mathrm{ml}^{-1}$, rhIL7 at $10 \mathrm{ng} \mathrm{ml}^{-1}$, rhIL25 at $10 \mathrm{ng} \mathrm{ml}^{-1}$, and rhIL33 at $0.5 \mathrm{ng} \mathrm{ml}^{-1}$ ). Recombinant human TL1A was used at $100 \mathrm{ng} \mathrm{ml}^{-1}$ for ILC2 restimulation. Anti-human DR3 (JD3, conjugated with $\mathrm{PE}$ ) was used to detect DR3 expression by flow cytometry.

The mouse ILC2s were sorted from single-cell suspension of mLN or lung as $\mathrm{Lin}^{-} \mathrm{sca}^{+}{ }^{+} \mathrm{CD} 127^{+} \mathrm{ST} 2^{+}$cells. ${ }^{37}$ Lineage markers used are biotin-conjugated antibodies for CD3 (CD3 $€, 145-2 \mathrm{C} 11)$, CD4 (RM4-5), CD5 (53-7.3), CD8, CD19 (6D5), CD11c (HL3), CD11b (M1/70), B220 (RA3-6B2), F4/80 (BM8), Ter119, IgM (11/41), IgE (R35-72), NK1.1 (PK136), DX5 (DX5), TCR $\gamma \delta$ (GL3), and Gr-1 (RB6-8C5). The following antibodies were also used for flow cytometry in an appropriate combination: anti-Sca-1 (D7; conjugated with PerCP-Cy5.5), anti-CD127 (A7R34; conjugated with PE or allophycocyanin (APC)), anti-c-Kit (2B8, conjugated with APC-H7), anti-ST2 (DJ8; conjugated with FITC; MD Biosciences, St Paul, MN), anti-CD287 (C398.4A; conjugated with APC), anti-KLRG1 (2F1; conjugated with FITC or PE), anti-mouse TNFR1 (55R-286; conjugated with APC), affinity purified anti-mouse DR3 (R\&D Systems), donkey-anti-goat secondary antibody (PE-conjugated; Rockland Immunochemicals, Gilbertsville, PA), and Streptavidin (conjugated to FITC, PE, or APC). Sorted cells were cultured in cRPMI 1640 media (RPMI-1640 media with $10 \%$ fetal bovine serum, glutamine, penicillin/streptomycin, and 2-mercaptoethanol (55 $\mu \mathrm{M}, \mathrm{Gibco})$, containing rhIL2 $\left(50 \mathrm{ng} \mathrm{ml}^{-1}\right)$, rmIL7 $\left(10 \mathrm{ng} \mathrm{ml}^{-1}\right)$, and rmIL33 $\left(100 \mathrm{ng} \mathrm{ml}^{-1}\right)$ for 5 days. Cells were rested in minimal cytokines (rmIL2 and rmIL7 only, each at $10 \mathrm{ng} \mathrm{ml}^{-1}$ ) for 2 days before they were used for experiments. For restimulation, 
suboptimal levels of cytokines were used (rmIL2 at $10 \mathrm{ng} \mathrm{ml}^{-1}, \mathrm{rmIL} 7$ at $10 \mathrm{ng} \mathrm{ml}^{-1}, \operatorname{rmIL} 25$ at $10 \mathrm{ng} \mathrm{ml}^{-1}$, and $\operatorname{rmIL33}$ at $0.5 \mathrm{ng} \mathrm{ml}^{-1}$ ). Recombinant murine TL1A was used at $100 \mathrm{ng} \mathrm{ml}^{-1}$ for ILC2 restimulation.

Microarray. ILC2s $\left(\mathrm{Lin}^{-} \mathrm{CRTH} 2{ }^{+} \mathrm{CD} 161^{+} \mathrm{CD} 127^{+}\right.$) were purified from human blood of five different donors by flow cytometry. The ILC2 yield ranged from 20,000 to 165,000 cells per donor $(0.001-$ $0.008 \%$ white blood cells). Purified ILC2s were expanded in vitro in the presence of rhIL2, rhIL7, rhIL33, and rhIL25 (each at $50 \mathrm{ng} \mathrm{ml}^{-1}$ ) for 7-10 days. Expanded cells maintained the ILC2 phenotype (Lin ${ }^{-}$ $\mathrm{CRTH} 2{ }^{+} \mathrm{CD} 161{ }^{+} \mathrm{CD} 127^{+}$). For transcriptional analysis, expanded cells were rested for 2 days in the presence of $1 \mathrm{ng} \mathrm{ml}^{-1}$ rhIL2 and rhIL7. For stimulations, we treated cells with media control, rhIL25 $\left(50 \mathrm{ng} \mathrm{ml}^{-1}\right)$, rhIL33 $\left(50 \mathrm{ng} \mathrm{ml}^{-1}\right)$ and both cytokines in combination for $6 \mathrm{~h}$. Whole RNA was isolated via RNeasy kit (Qiagen, Hilden, Germany) and subjected to microarray analysis with Agilent chip (Santa Clara, CA). Accession number is GSE 45458.

Real-time PCR. RNA was isolated from in vitro expanded human ILC2s from five different donors or sorted murine MLN ILC2s with an RNeasy mini kit (Qiagen). An ABI 7500 Real-Time PCR system (Applied Biosystems) was used for real-time RT-PCR. Primers for human DR3, human TNFR1, murine GATA3, murine amphiregulin, murine IL17RB, murine ST2, and murine IL13 are all from ABI (Life Technologies). Data were normalized to results obtained for ribosomal protein L19, ${ }^{38}$ and are presented as $2^{-\Delta C t}$.

Ileum organ culture. Ileum was removed, cut open longitudinally, and washed in PBS containing penicillin and streptomycin. The ileum was then placed in 24-well flat-bottom tissue culture plate containing $1 \mathrm{ml}$ of fresh RPMI-1640 with $1 \%$ penicillin and streptomycin and incubated at $37^{\circ} \mathrm{C}$ for $24 \mathrm{~h}$. Culture supernatants were harvested and assayed for cytokines.

Cytokine injections. For intraperitoneal injections, rmTL1A $(5 \mu \mathrm{g})$, rmIL25 (500 ng), or rmIL33 (500 ng) were administrated as indicated. PBS was used as control. The mLNs were collected for FACS and serum were collected for cytokine analysis. For intranasal administration, $10 \mu \mathrm{g}$ of rmTL1A was used. Peritoneal lavage was collected by flushing the abdominal cavity with $1 \mathrm{ml}$ PBS for cytokine analysis, or with $4 \mathrm{ml}$ of PBS for cell enumeration and FACS. BALF was performed by flushing out the lung with $1.5 \mathrm{ml}$ PBS via a tracheal cannula, and lung lymphocytes were isolated after collagenase $\mathrm{D}$ digestion. The cells and BALF were then subjected to FACS and/or cytokine analysis.

Cytokine assays. Intracellular detection of IL5 and IL13 was performed using the BD Cytofix/Cytoperm Fixation/Permeabilization Kit (BD Pharmingen) following the manufacturer's protocol with the following antibodies: PE-IL5 (clone TRFK5, eBioscience) and AF647IL13 (clone eBio13A, eBioscience). Cytokine concentrations were measured in cell culture supernatants with ELISA kit (eBioscience) or with Bio-plex beads (Bio-Rad, Hercules, CA) and subsequent detection in Luminex 100 system according to the manufacturer's recommended protocol (Luminex, Austin, TX).

Intranasal papain challenge. Groups of mice were anesthetized with isoflurane and exposed intranasally to $25 \mu \mathrm{g}$ papain (Wako, Richmond, VA) in $50 \mu \mathrm{l}$ PBS on days 0,3 , and 6 . On day 7 , BAL was performed by flushing out the lung with $1.5 \mathrm{ml}$ PBS via a tracheal cannula. Diced lung fragments were digested in buffer containing collagenase $\mathrm{D}$ for $30 \mathrm{~min}$ at $37^{\circ} \mathrm{C}$. Lung homogenate was obtained by meshing through a $40-\mu \mathrm{m}$ cell strainer and the cells were further purified using a $37.5 \%$ percoll gradient. Cells were stained for surface antigens.

$\boldsymbol{N}$. brasiliensis infection. Infective, third-stage larvae of $N$. brasiliensis (L3) (specimens on file at the US National Parasite Collection, US National Helminthological Collection, Collection 81930, Beltsville,
$\mathrm{MD)}$ were propagated and stored at room temperature in fecal/ charcoal/peat moss culture plates until use. Then, 8-10-week-old $\mathrm{Dr}^{-1-}$ mice and $\mathrm{Dr} 3^{+/+}$WT littermates were infected subcutaneously with $500 \mathrm{~N}$. brasiliensis L3 larvae in $200 \mu \mathrm{l}$ of saline on the flank. Animals were placed on polymixin $b$ and neomycin-medicated water for 5 days after infection. When indicated, each mouse was given $200 \mu \mathrm{g}$ antibodies i.p. every 2 days starting 1 day before infection. Mice were killed and studied at day 7 or day 10 after infection. The mLNs, BALF, lungs, and serum were harvested 7 days after infection. At day 10 after infection, adult worms were detected quantitatively by incubating the opened small intestine in $1 \times$ Hanks' Balanced Salt Solution (HBSS; Gibco) at $37^{\circ} \mathrm{C}$ for $1 \mathrm{~h}$ and counting under a microscope. In general, WT mice completely expelled worms by day 10 after inoculation, and therefore, the presence of adult worms in the intestine indicates delayed expulsion.

Histology. Tissue samples were fixed in formalin and processed to paraffin blocks. Sections $(4 \mu \mathrm{m})$ were stained with hematoxylin and eosin. Pulmonary lesions were scored for inflammation, granuloma formation, and airway goblet cell hyperplasia. Aggregate lesion severity scores from 0 to 10 were determined for each sample and used for groupwise comparison of score means.

Statistics. Statistical analyses were performed using JMP version 8.0.2 software (SAS Institute, San Francisco, CA). We made comparisons for each pair with Student's $t$-test, with $P$-values $<0.05$ considered significant.

SUPPLEMENTARY MATERIAL is linked to the online version of the paper at http://www.nature.com/mi

\section{ACKNOWLEDGMENTS}

ST2-deficient mice were kindly provided by Andrew McKenzie at the Medical Research Council. We thanks Merone Roose-Girma for generation of TL1A transgenic mice; Jason de Voss in Translational Immunology for preliminary characterization of the transgenic mice; Sarah Hymowitz, Michelle W. Lee, and Hongkang Xi for protein advice and purifications; Chauncey Spooner for ILC2 isolation; Alex Abbas for microarray annotation; and members of FACS Lab for cell sorting and Luminex analysis. We thanks Wenjun Ouyang, Ira Mellman, Harinder Singh, Chauncey Spooner, and Andrew C. Chan for advice on the manuscript.

\section{AUTHOR CONTRIBUTIONS}

$X . Y$. designed and contributed to all experiments and prepared the manuscript; R.P. and V. R-C. generated ILC2 microarray data; J.Z. performed cytokine injection studies; W.P.L. designed and supervised the in vivo studies; D.Y. and M.X. designed and performed helminth studies; P.C. performed pathology and IHC; J.L.G. initiated and supervised the project, and wrote the manuscript.

\section{DISCLOSURE}

All authors, during these studies, were employed by Genentech that develops and markets drugs for profit.

co 2014 Society for Mucosal Immunology

\section{REFERENCES}

1. Spits., H. \& Cupedo, T. Innate lymphoid cells: emerging insights in development, lineage relationships, and function. Annu. Rev. Immunol. 30, 647-675 (2012).

2. Spits, H. et al. Innate lymphoid cells-a proposal for uniform nomenclature. Nat. Rev. Immunology 13, 145-149 (2013).

3. Chang, Y.J. et al. Innate lymphoid cells mediate influenza-induced airway hyper-reactivity independently of adaptive immunity. Nat. Immunol. 12, 631-638 (2011).

4. Monticelli, L.A. et al. Innate lymphoid cells promote lung-tissue homeostasis after infection with influenza virus. Nat. Immunol. 12, 1045-1054 (2011). 
5. Moro, K. et al. Innate production of $\mathrm{T}(\mathrm{H}) 2$ cytokines by adipose tissueassociated c-Kit(+)Sca-1(+) lymphoid cells. Nature 463, 540-544 (2010).

6. Neill, D.R. et al. Nuocytes represent a new innate effector leukocyte that mediates type-2 immunity. Nature 464, 1367-1370 (2010).

7. Saenz, S.A. et al. IL25 elicits a multipotent progenitor cell population that promotes $T(H) 2$ cytokine responses. Nature 464, 1362-1366 (2010).

8. Price, A.E. et al. Systemically dispersed innate IL-13-expressing cells in type 2 immunity. Proc. Natl. Acad. Sci. USA 107, 11489-11494 (2010).

9. Yasuda, K. et al. Contribution of IL-33-activated type Il innate lymphoid cells to pulmonary eosinophilia in intestinal nematode-infected mice. Proc. Natl. Acad. Sci. USA 109, 3451-3456 (2012).

10. Vonarbourg, C. \& Diefenbach, A. Multifaceted roles of interleukin-7 signaling for the development and function of innate lymphoid cells. Semin. Immunol. 24, 165-174 (2012).

11. Fort, M.M. et al. IL-25 induces IL-4, IL-5, and IL-13 and Th2-associated pathologies in vivo. Immunity 15, 985-995 (2001).

12. Fallon, P.G. et al. Identification of an interleukin (IL)-25-dependent cell population that provides IL-4, IL-5, and IL-13 at the onset of helminth expulsion. J. Exp. Med. 203, 1105-1116 (2006).

13. Hoshino, K. et al. The absence of interleukin 1 receptor-related T1/ST2 does not affect Thelper cell type 2 development and its effector function. $J$. Exp. Med. 190, 1541-1548 (1999).

14. Hardman, C.S., Panova, V. \& McKenzie, A.N. IL-33 citrine reporter mice reveal the temporal and spatial expression of IL-33 during allergic lung inflammation. Eur. J. Immunol. 43, 488-498 (2012).

15. Kim, B.S. et al. TSLP elicits IL-33-independent innate lymphoid cell responses to promote skin inflammation. Sci. Transl. Med. 5, 170ra116 (2013).

16. Meylan, F, Richard, A.C. \& Siegel, R.M. TL1A and DR3, a TNF family ligand-receptor pair that promotes lymphocyte costimulation, mucosal hyperplasia, and autoimmune inflammation. Immunol. Rev. 244, 188-196 (2011).

17. Yamazaki, K. et al. Single nucleotide polymorphisms in TNFSF15 confer susceptibility to Crohn's disease. Hum. Mol. Genet. 14, 3499-3506 (2005).

18. Franke, A. et al. Genome-wide meta-analysis increases to 71 the number of confirmed Crohn's disease susceptibility loci. Nat. Genet. 42, 1118-1125 (2010).

19. Meylan, F. et al. The TNF-family cytokine TL1A drives IL-13-dependent small intestinal inflammation. Mucosal Immunol. 4, 172-185 (2011).

20. Shih, D.Q. et al. Constitutive TL1A (TNFSF15) expression on lymphoid or myeloid cells leads to mild intestinal inflammation and fibrosis. PLoS One 6 , e16090 (2011).

21. Taraban, V.Y. et al. Sustained TL1A expression modulates effector and regulatory T-cell responses and drives intestinal goblet cell hyperplasia. Mucosal Immunol. 4, 186-196 (2011).

22. Jin, S. et al. TL1ATTNFSF15 directly induces proinflammatory cytokines, including TNFalpha, from CD3 + CD161 + T cells to exacerbate gut inflammation. Mucosal Immunol. 6, 886-899 (2012).
23. Scanlon, S.T. \& McKenzie, A. Type 2 innate lymphoid cells: new players in asthma and allergy. Curr. Opin. Immunol. 24, 707-712 (2012).

24. Mjosberg, J. et al. The transcription factor GATA3 is essential for the function of human type 2 innate lymphoid cells. Immunity 37, 649-659 (2012).

25. Hoyler, T. et al. The transcription factor GATA-3 controls cell fate and maintenance of type 2 innate lymphoid cells. Immunity 37, 634-648 (2012).

26. Maizels, R.M., Hewitson, J.P. \& Smith, K.A. Susceptibility and immunity to helminth parasites. Curr. Opin. Immunol. 24, 459-466 (2012).

27. Halim, T.Y., Krauss, R.H., Sun, A.C. \& Takei, F. Lung natural helper cells are a critical source of Th2 cell-type cytokines in protease allergen-induced airway inflammation. Immunity 36, 451-463 (2012).

28. Saenz, S.A., Taylor, B.C. \& Artis, D. Welcome to the neighborhood: epithelial cell-derived cytokines license innate and adaptive immune responses at mucosal sites. Immunol. Rev. 226, 172-190 (2008).

29. Grogan, J.L. \& Ouyang, W. A role for Th17 cells in the regulation of tertiary lymphoid follicles. Eur. J. Immunol. 42, 2255-2262 (2012).

30. Kim, M.Y. et al. Neonatal and adult CD4 + CD3- cells share similar gene expression profile, and neonatal cells up-regulate OX40 ligand in response to TL1A (TNFSF15). J. Immunol. 177, 3074-3081 (2006).

31. Fang, L, Adkins, B, Deyev, V \& Podack, E.R. Essential role of TNF receptor superfamily 25 (TNFRSF25) in the development of allergic lung inflammation. J. Exp. Med. 205, 1037-1048 (2008).

32. Meylan, F. et al. The TNF-family receptor DR3 is essential for diverse T cell-mediated inflammatory diseases. Immunity 29, 79-89 (2008).

33. Pappu, B.P. et al. TL1A-DR3 interaction regulates Th17 cell function and Th17-mediated autoimmune disease. J. Exp. Med. 205, 1049-1062 (2008).

34. Ni, J, Nolte, B, Arnold, A., Fournier, P. \& Schirrmacher, V. Targeting anti-tumor DNA vaccines to dendritic cells via a short CD11c promoter sequence. Vaccine 27, 5480-5487 (2009).

35. Tang, T. et al. A mouse knockout library for secreted and transmembrane proteins. Nat. Biotechnol. 28, 749-755 (2010).

36. Mjosberg, J.M. et al. Human IL-25- and IL-33-responsive type 2 innate lymphoid cells are defined by expression of CRTH2 and CD161. Nat. Immunol. 12, 1055-1062 (2011).

37. Pishdadian, A, Varasteh, A.R. \& Sankian, M. Type 2 innate lymphoid cells: friends or foes-role in airway allergic inflammation and asthma. J. Allergy 2012, 130937 (2012).

38. Yu, X. et al. The surface protein TIGIT suppresses T cell activation by promoting the generation of mature immunoregulatory dendritic cells. Nat. Immunol. 10, 48-57 (2009).

(c)(1) $\odot \odot$ This work is licensed under the Creative Commons At 1 aribution-NonCommercial-No Derivative Works 3.0 Unported License. To view a copy of this license, visit http://creativecommons.org/licenses/by-nc-nd/3.0/ 